tertiary hospital, based on available microbiology results. The deescalation suggestion was made through the electronic prescribing software. The variables analysed were: number of patients prescribed carbapenems, prescribing speciality, request for cultures, microorganisms isolated and interventions performed. De-escalations carried out without pharmacy intervention were also assessed.

Results Total number of prescriptions was 433. The most prescribed carbapenem was ertapenem (37.6\%) followed by meropenem $(36 \%)$. The carbapenem most used in Internal Medicine was meropenem (58.2\%) and in Urology, imipenem (75\%). Ertapenem was used more frequently in General Surgery (53.7\%) and Vascular Surgery (86.0\%). Out of a total of 316 requested tests, 172 (54.4\%) were positive. The most common pathogen isolated was Escherichia Coli (24.7\%) $20.8 \%$ of which were Extended-Spectrum BetaLactamase (ESBL)-producing, $60 \%$ of which were sensitive to piperacillin-tazobactam or fosfomycin. Klebsiella spp. were isolated in $3.6 \%$, of which $33.3 \%$ were ESBL-producing and $50 \%$ were sensitive to piperacillin-tazobactam. Total treatments subject to de-escalation were $96(55.8 \%)$, out of 172 showing this possibility, where $74(77.1 \%)$ were carried out by initiatives of medical teams and $22(22.9 \%)$ after pharmacy interventions. The Services with a higher degree of acceptance of pharmacy interventions were Internal Medicine (36.4\%) and General Surgery (27.3\%).

Conclusions Although the therapeutic de-escalation of empirical treatments with carbapenems was a low percentage, nevertheless pharmacy interventions achieved an increase of this practise, with the more receptive specialties being Internal Medicine and General Surgery.

No conflict of interest.

\section{OHP-021 DEVELOPMENT AND IMPLEMENTATION OF A PERIPHERAL STANDARD PARENTERAL NUTRITION FOR A NEONATOLOGY DEPARTMENT}

doi:10.1136/ejhpharm-2013-000276.395

A Belaustegi Foronda, B Sordo Aisa, Z Baskaran Kaltzakorta, A De Basagoiti Gorordo, I Bilbao Meseguer, J Hernandez Goicoechea, B San Jose Ruiz, M Castaño Lopez, E Rodriguez España, S Sautua Larreategi. Hospital Universitario de Cruces, Pharmacy, Bilbao, Spain

Background Parenteral nutrition (PN) for neonates has to be infused by a central line, due to the high osmolarity resulting from the recommended requirements. The central catheter frequently needs to be removed, and therefore PN may have to be administered by a peripheral line. This problem has been resolved by infusion of enriched glucose solutions, minus the protein input, which is very important in order to avoid catabolism.

Purpose To develop a standard PN with glucose, electrolytes and amino acids, suitable for peripheral infusion and available for the Neonatology department at any time.

The aim is to infuse $100 \mathrm{~mL} / \mathrm{Kg}$.

Materials and Methods We performed a literature search about standard PN and we made microbiological and biochemical cheques to ensure the stability and integrity of the solution, after keeping it refrigerated for seven days.

Results We developed a standard PN solution with the following composition per $100 \mathrm{~mL}$ :

Amino acids: $2 \mathrm{~g}$

Glucose: $9.5 \mathrm{~g}$

Sodium: $4 \mathrm{mEq}$

Potassium: $2 \mathrm{mEq}$

Magnesium: $0.2 \mathrm{mEq}$

Calcium: $1.5 \mathrm{mEq}$

Phosphate: $0.8 \mathrm{mmol}$

Osmolarity: $792 \mathrm{mOsm} / \mathrm{L}$

Total calories: $46 \mathrm{Kcal}$
Weekly, we prepare four $500 \mathrm{ml}$ bags from a stock solution. We give the neonatology department two so they can hold a small stock and we keep the other two in order to cheque when we need to make another batch.

From implementation, in February 2012, the microbiological controls have always been negative and the biochemical controls have demonstrated that degradation does not occur after refrigeration for seven days.

Conclusions This formulation makes it possible for the physicians to continue with the nutritional support, by peripheral infusion, at any time.

However this type of nutritional solutions is only suitable for meeting the nutritional requirements for short periods, until a new central catheter is placed or the neonate is able to have complete enteral feeding.

No conflict of interest.

\section{OHP-022 DEVELOPMENT OF AN AUTOMATIC METHOD FOR THE COMPARISON OF MASKS USED IN 81MKR/99MTC DUAL ISOTOPE PLANAR VENTILATION/PERFUSION SCINTIGRAPHY}

doi:10.1136/ejhpharm-2013-000276.396

${ }^{1} \mathrm{H}$ De Blander, ${ }^{1} \mathrm{~A}$ Maulois, 'E Camps, 'B Bonan, ${ }^{2} \mathrm{E}$ Le Stanc, ${ }^{2} \mathrm{D}$ Vilain. ${ }^{1}$ Hopital Foch, Service of Pharmacy, Suresnes, France; ${ }^{2}$ Hopital Foch, Service of Nuclear Medicine, Suresnes, France

Background Various pulmonary diseases can be evaluated by ventilation and perfusion scintigraphy with a continuous inhalation of $81 \mathrm{mKrypton}$. Leak of radionuclide during inhalation is a major issue for image quality and requires the intervention of a technician, exposing him-her to gamma radiation from the patient.

Purpose To compare two masks: the DAR breathing system (Covidien) and the Performa Trak VNI (Philips Respironics) including a harness for ventilation scintigraphy and to develop an automatic method for evaluating the quality of $81 \mathrm{mkr}$ inhalation.

Materials and Methods We enrolled and randomised 48 patients to breathe through two types of masks: DAR $(n=25)$ or VNI $(\mathrm{n}=23)$. After intravenous injection of $99 \mathrm{mTc}$-labelled macroaggregated human albumin (LyoMAA, Covidien) and during continuous inhalation of $81 \mathrm{mKr}$ extracted from a $81 \mathrm{Rb}-81 \mathrm{mKr}$ generator (Kryptoscan, Covidien), eight incidences were acquired on a dualhead gamma camera. Three parameters were automatically computed by an automatic segmentation method: the mean ventilation counts (mcounts), an index of constancy of the inhalation rates (Cvent/perf) reflecting variations of the ventilation counts [(maximum-minimum)/median] between incidences compared to perfusion and an index of inhalation leak $(\mathrm{mBN}$, the maximum background noise mean on the profiles incidences). Non-parametric tests of comparison of variance and proportion were used (Mood test and Fisher exact Test).

Results Variance of Cvent/perf and $\mathrm{mBN}$ were significantly higher $(\mathrm{P}=0.03)$ in the DAR group. In this group $6 / 25(24 \%)$ patients had parameters out of the distribution of the VNI group and $11 / 25$ (44\%) needed the help of a technician to hold their mask. No difference in the mcounts rate was observed.

Conclusions Index of variability of the ventilation rate as well as background noise were significantly higher in the DAR group and involved about $24 \%$ of the patients. Support with VNI masks improved the image quality, decreased exposure to radiation and guaranteed constancy in care compared to DAR. Nevertheless high costs restrict their use.

No conflict of interest. 